\title{
Opinión
}

\section{Abordaje de la enfermedad arterial coronaria Reflexión}

\section{(An approach to coronary artery disease)}

\author{
Oswaldo Gutiérrez- Sotelo
}

\section{La enfermedad más común del mundo}

La arteriosclerosis coronaria y de otros territorios vasculares se inicia desde la infancia, condicionada por determinantes genéticos muy prevalentes en la población ${ }^{1}$ : alteraciones en el metabolismo de la sal, de la glucosa y de los lípidos, sazonados con factores ambientales como hábitos alimentarios, de ejercicio, fumar tabaco, cocinar con leña y de "estrés", lo que termina dando por resultado la aparición de placas dentro de la luz arterial, cuyo contenido es básicamente lípidos y células inflamatorias, las cuales secretan sustancias que tienen propiedades vasoconstrictoras, proliferativas y procoagulantes.

A lo largo de la vida, la placa va creciendo en 2 modalidades: una lenta y progresiva, a partir de mayor acumulación de lípidos y proliferación celular. La otra, subitus suscipio: la pared arterial es de naturaleza elástica y la placa no, por lo tanto en algún momento esta se fractura, exponiendo elementos del interior de la placa que no suelen tener contacto con la sangre circulante; en respuesta a ello se genera un trombo endoluminal, con lo cual se reduce la luz arterial bruscamente en un determinado porcentaje o causando oclusión total. ${ }^{2}$

La aparición clínica de la enfermedad, después de décadas de iniciado el proceso, tiene 2 modalidades: los síntomas que se originan en la obstrucción progresiva y ahora avanzada de la luz arterial y aquellos síntomas determinados por una súbita trombosis que obstruye rápida y significativamente la luz arterial.

La reducción crónica de la luz arterial condiciona síntomas solo cuando se supera la capacidad del vaso de aportar sangre al tejido, tal como ocurre con la mayor demanda de oxígeno del miocardio que produce el ejercicio físico: el dolor resultante de la isquemia miocárdica es la angina de pecho. Este tipo de evolución indica que el organismo ha ejecutado mecanismos compensatorios que permiten que el paciente no tenga prácticamente ningún síntoma en reposo, a pesar de tener obstrucciones luminales en su árbol arterial coronario.

Cardiólogo, Electrofisiólogo

Correspondencia: Hospital Clínica Bíblica,Av I4, San José, Costa Rica Apdo. Postal 47I-I300, email: oswcr@hotmail.com

ISSN 0001-6002/2008/50/3/172-174

Acta Médica Costarricense, $\mathbb{C} 2008$

Colegio de Médicos y Cirujanos
En las extremidades inferiores en las que exista el mismo problema obstructivo, tal dolor impide continuar el ejercicio, condición denominada "claudicación intermitente", puesto que desaparece con el reposo.

Por otro lado, la disminución brusca y muchas veces total de la circulación coronaria por causa de una trombosis aguda condiciona un síndrome coronario agudo que va desde el dolor anginoso intenso y de reposo, con o sin necrosis miocárdica, hasta la muerte súbita cardiaca. El dolor anginoso de reposo sin necrosis se llama "angina inestable" o "síndrome coronario agudo sin elevación del segmento ST", mientras que cuando se acompaña del proceso necrótico se llama "infarto agudo del miocardio" o "síndrome coronario agudo con elevación del segmento ST", nomenclatura útil para tomar la decisión en la sala de urgencias de administrar fibrinolíticos como la estreptokinasa en pacientes del segundo grupo. En ambos, la extensión del territorio isquémico en riesgo o en curso de necrosis, determina el pronóstico intrahospitalario y en el largo plazo. En cambio, la muerte súbita cardiaca se debe a la aparición de fibrilación ventricular y colapso hemodinámico, condicionada por un grave desarreglo de la electrofisiología del miocardio, secundaria a su vez a una extensa área isquémica, causada por la trombosis intracoronaria aguda. En todos estos casos agudos, la falta de tiempo para la ejecución de mecanismos compensatorios produce cuadros agudos que descompensan gravemente al paciente y que causan muchas muertes.

En extremidades inferiores la obstrucción arterial aguda condiciona dolor isquémico con frialdad y palidez, que de no resolverse, conducirá a la necrosis y pérdida de la extremidad; en el sistema nervioso central, diversos cuadros isquémicos agudos se denominan en conjunto "ataque vascular cerebral" y también en algunos casos se debe administrar fibrinolíticos para evitar llegar a la necrosis y el infarto cerebral.

\section{¿Dónde empieza el abordaje de este grave problema de salud pública?}

La palabra más importante es PREVENCIÓN. No solo las personas con historia familiar de diabetes, hipertensión, hiperuricemia y cardiopatías deben aprender a consumir los alimentos que en cantidades inadecuadas pueden acelerar la aparición fenotípica de estas condiciones. Todos deben además conocer sus valores bioquímicos con cierta 
regularidad a pesar de ser muchas veces sujetos jóvenes y totalmente asintomáticos, pues está bien establecido el valor de la terapia preventiva, por ejemplo, con estatinas. La suspensión del hábito de fumar y el ejercicio regular tienen también beneficios bien documentados. ${ }^{3}$

La segunda palabra más importante es METAS. En los pacientes hipertensos, diabéticos y dislipidémicos deben alcanzarse las metas terapéuticas establecidas por los paneles de expertos mundiales: presión arterial sistólica/diastólica igual o menor de 130/80 mmHg, colesterol LDL menor de $100 \mathrm{mg} / \mathrm{dL}$ y Hb A1c menor de 6,5\%. A pesar de que ello es relativamente fácil de alcanzar desde un punto de vista técnico, menos de la mitad de estos pacientes las alcanza, incluso en países desarrollados, en parte debido a que los médicos no educamos y motivamos a estos pacientes a ser partícipes directos y activos de la solución de su condición. ${ }^{4}$

El medicamento "preventivo" más popular para la trombosis coronaria -el gran temor en este grupo de pacientes- es la aspirina, pero dado que tiene algunos efectos indeseables, no debe utilizarse de manera indiscriminada. En ausencia de cualquier signo que indique que ya existe enfermedad vascular clínicamente comprobable, solo es recomendada para personas con los factores de riesgo señalados, incluyendo la historia familiar.

\section{Paciente con enfermedad vascular manifiesta}

En el paciente con un síndrome vascular crónico como la angina crónica de esfuerzo, la terapia tiene que ser también preventiva, pues el objetivo es detener el avance del proceso $\mathrm{y}$, sobre todo, disminuir la inflamación para prevenir la aparición de un evento vascular agudo. Esto se logra con fármacos antihipertensivos e hipolipemiantes, ejercicio $\mathrm{y}$ control metabólico. En principio, estos pacientes no deben someterse a revascularización de sus lesiones obstructivas con prótesis endoluminales o "stent" coronarios, porque el trauma del procedimiento no hace sino aumentar la inflamación de la placa intervenida $y$, por ende, incrementar la posibilidad de una trombosis aguda que hasta el momento no existía. Los pacientes con angina crónica solo se deben revascularizar cuando los síntomas impiden su vida normal o cuando se demuestre objetivamente que el territorio isquémico es tan extenso como para elevar la probabilidad de muerte cardiaca. Esto se sabe con las denominadas pruebas funcionales, como la de esfuerzo, o el ecocardiograma de esfuerzo. Si las lesiones son múltiples e incluyen el tronco principal o la principal rama de la arteria coronaria izquierda, la terapia de revascularización por elegir son los puentes coronarios con arteria torácica interna o con vena safena, el bypass coronario. Este procedimiento no aumenta la infamación endoluminal, pero el hecho de requerir circulación extracorpórea implica cierta morbilidad y mortalidad que deben ponderarse, según cada caso.,

\section{Paciente con un síndrome coronario agudo}

Después de administrar fibrinolíticos a quienes lo ameritan y estabilizarse el paciente con algunos días de reposo y terapia farmacológica ${ }^{7,8}$ se valoran las condiciones del territorio isquémico o necrótico con una prueba funcional, y si existen criterios de riesgo, se revasculariza con stent o bypass, según la anatomía coronaria. De lo contrario, se reinicia la prevención. La intervención coronaria en pleno evento agudo solo se reserva para los casos cuando está amenazada la vida o gran parte de la viabilidad del miocardio, que se justifica pese al aumento de la inflamación en la placa, con la potencial reoclusión que podría presentarse. Esto requiere una logística e infraestructura hospitalaria bien organizada.

$\mathrm{Y}$ ¿qué se debe hacer con los pacientes que han sobrevivido a una muerte súbita? (que son los menos); además de valorarlos funcionalmente para determinar si requieren o no revascularización coronaria, en general, si tienen deterioro significativo de la contractilidad ventricular, se debe colocar un cardiodesfibrilador implantable, terapia que ha demostrado disminuir contundentemente el elevado riesgo de muerte de estos pacientes. ${ }^{9}$

\section{El problema no solo compete a los especialistas}

A los cardiólogos hemodinamistas les corresponde realizar la angioplastía coronaria percutánea y a los cardiólogos electrofisiólogos, la colocación de cardiodesfibriladores; a los cirujanos cardiovasculares les compete hacer la cirugía de bypass y a los cardiólogos no intervencionistas, las pruebas funcionales e indicar el tipo de revascularización, pero a todos ellos les apremia trabajar en prevención, al igual que a los médicos generales y especialistas no cardiólogos. A la comunidad no médica (periodistas, maestros, padres de familia) le compete la educación en cuanto a hábitos alimentarios, ejercicio e información preventiva, comenzando, desde luego, con el ejemplo. Se dispone de suficientes recursos humanos para la gran tarea preventiva, que es por cierto la más barata, en términos de costo por persona (Figura 1). ¿Por qué entonces esta enfermedad es cada vez más prevalente y el promedio de edad de los afectados cada vez menor? El factor cultural, que incluye estilos de vida no saludables, desinterés por informarse, consumismo y menosprecio por los recursos que se destinan al manejo de este problema, es el principal escollo. La población teme a la enfermedad coronaria, pero a veces hace muy poco para prevenirla; conoce de la cirugía de bypass y de los stents coronarios pero no sabe que ambas son costosas terapias paliativas, no preventivas $\mathrm{y}$, mucho menos, curativas. O sea, se quiere y se conoce el buen sistema de bomberos que apaga los incendios, pero no se revisan las instalaciones eléctricas ni se deja de jugar con fuego para prevenirlos, de la misma forma que preocupa el calentamiento global, pero se usa el automóvil en lugar de caminar (cuando se pueda) y así sucesivamente. El problema es mundial.

Sin embargo, también hay logros: en casi todos los países fumar se ha vuelto complicado para quienes tienen el hábito; la información, antes poco accesible, hoy es fácilmente alcanzable y se dispone de potentes medicamentos que han hecho más fácil y seguro alcanzar las metas terapéuticas. Pero, al igual que en los asuntos comerciales, hay un gran segmento de la población mundial que está en desventaja para competir por buenos servicios de salud y por información, que tal vez nunca en su vida tocará una computadora personal y seguirá cocinando con leña. 


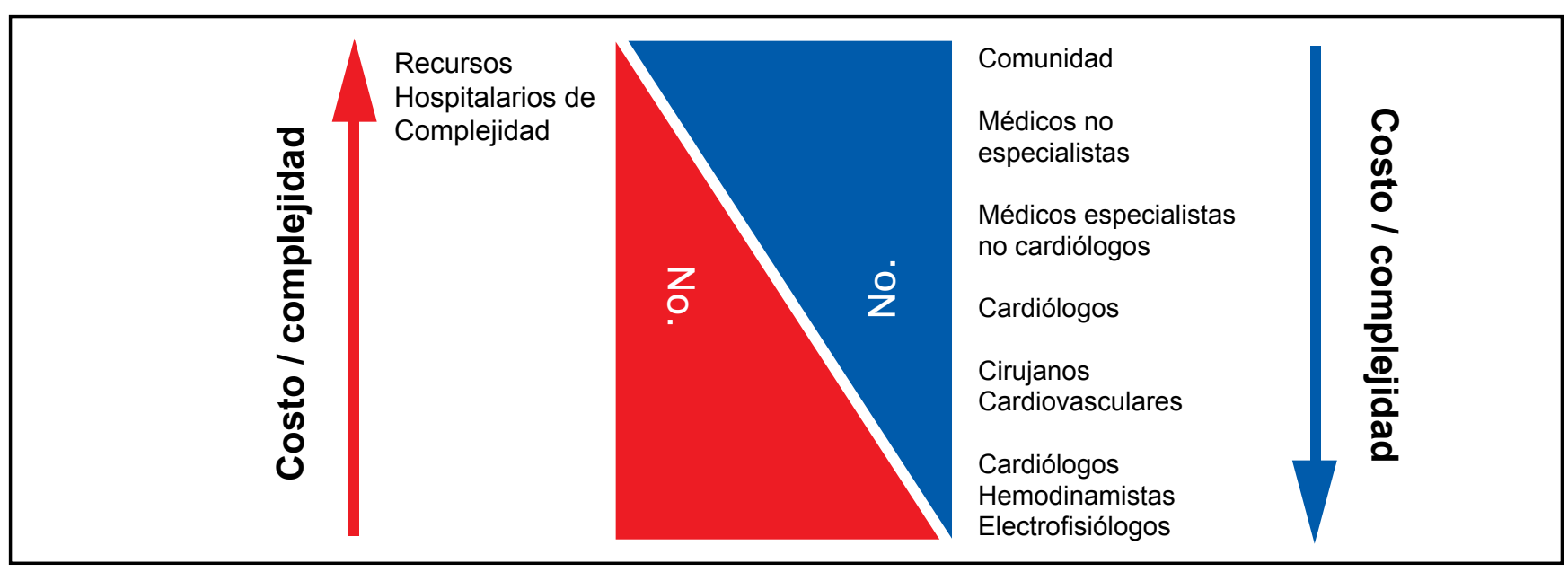

Figura. 1. El triángulo rojo representa la proporción entre la cantidad disponible de recursos hospitalarios de complejidad para el manejo de la enfermedad cardiovascular y los recursos educativos para su prevención: escasos los primeros en el vértice y accesibles y económicos los segundos en la base. El triángulo azul representa la proporción entre la cantidad de personas disponibles para la prevención y educación cardiovascular y la de personas con alto grado de especialización para el tratamiento de las formas avanzadas de la enfermedad: abundantes en la base y escasos y de alto costo en el vértice.

A diferencia de los avances en la prevención de las enfermedades infecto-contagiosas, en la mayoría de países la arterioesclerosis coronaria y de otros territorios vasculares va en aumento, así como su costo, reflejo de los patrones culturales y de consumo. ${ }^{10} \mathrm{Al}$ igual que con los cambios climáticos y otros problemas graves como la inseguridad ciudadana, la prevención de este problema reside en la educación familiar y en el cultivo de buenos hábitos. Es tarea de todos.

\section{Referencias}

1. WHO global infobase. En: http://www.who.int/infobase/report.aspx?r $\mathrm{id}=115 \& \mathrm{iso}=\mathrm{CRI} \& \mathrm{dm}=5 \&$ searchButton $=$ Search + Surveys. Consultado el 9 de mayo de 2008

2. Gutstein DE, Fuster V. Pathophysiology and clinical significance of atherosclerotic plaque rupture. Cardiovascular Research 1999 41:323333. (texto completo en: http://cardiovascres.oxfordjournals.org/cgi/ content/full//41/2/323)

3. McGill HC, McMahan A, Gidding SS. Preventing Heart Disease in the 21 st Century. Implications of the Pathobiological Determinants of Atherosclerosis in Youth (PDAY) Study. Circulation 2008;117:12161227

4. Geller JC, Cassens S, Brosz M, Keil U, Bernarding J, Kropf S, Bierwirth RA, Lippmann-Grob B, Schultheiss HP, Schlüter K, Pels K. Achievement of guideline-defined treatment goals in primary care: the german coronary risk management (CoRiMa) study. Eur Heart J 2007; 28:3051-8.

5. Smith SC Jr, Feldman TE, Hirshfeld, JW Jr, Jacobs AK, Kern MJ, King SB III, Morrison DA, O’Neill WW, Schaff HV, Whitlow PL, Williams DO. ACC/AHA/SCAI 2005 guideline update for percutaneous coronary intervention: a report of the American College of Cardiology/American heart association task force on Practice Guidelines (ACC/AHA/SCAI Writing Committee to Update the 2001 Guidelines for Percutaneous Coronary Intervention). Circulation. 2006;113: e166-e286. Available at: http://www.americanheart.org

6. Eagle KA, Guyton RA, Davidoff R, Edwards FH, Ewy GA, Gardner TJ, Hart JC, Herrmann HC, Hillis LD, Hutter AM Jr, Lytle BW, Marlow RA, Nugent WC, Orszulak TA. ACC/AHA 2004 guideline update for coronary artery bypass graft surgery: a report of the
American College of Cardiology/American Heart Association. Task Force on Practice Guidelines (Committee to Update the 1999 Guidelines for coronary artery bypass graft surgery circulation 2004;110;e340-e437. Available at: http://www.acc.org/clinical/ guidelines/cabg/cabg.pdf.

7. J. L. Anderson, C. D. Adams, E. M. Antman, C. R. Bridges, R. M. Califf, D. E. Casey Jr, W. E. Chavey II, F. M. Fesmire, J. S. Hochman, T. N. Levin, et al. ACC/AHA 2007 Guidelines for the Management of Patients With Unstable Angina/Non-ST-Elevation Myocardial Infarction: A Report of the American College of Cardiology/American Heart Association Task Force on Practice Guidelines (Writing Committee to Revise the 2002 Guidelines for the Management of Patients With Unstable Angina/Non-ST-Elevation Myocardial Infarction) Developed in Collaboration with the American College of Emergency Physicians, the Society for Cardiovascular Angiography and Interventions, and the Society of Thoracic Surgeons Endorsed by the American Association of Cardiovascular and Pulmonary Rehabilitation and the Society for Academic Emergency Medicine. J. Am. Coll. Cardiol., August 14, 2007; 50(7): e1 - e157. http://circ. ahajournals.org/cgi/content/abstract/CIRCULATIONAHA.107. $185752 \mathrm{v} 1$

8. Antman EM, Anbe DT, Armstrong PW, Bates ER, Green LA, Hand M, Hochman JS, Krumholz HM, Kushner FG, Lamas GA, Mullany CJ, Ornato JP, Pearle DL, Sloan MA, Smith SC Jr. ACC/AHA guidelines for the management of patients with ST-elevation myocardial infarction: executive summary: a report of the ACC/AHA Task Force on Practice Guidelines (Committee to Revise the 1999 Guidelines on the Management of Patients With Acute Myocardial Infarction). J Am Coll Cardiol 2004;44: 671-719. http://www.acc.org/ qualityandscience/clinical/guidelines/STEMI/Guideline1/index.htm

9. Zipes DP, Camm AJ, Borggrefe M, Buxton AE, Chaitman B, Fromer M, Gregoratos G, Klein G, Moss AJ, Myerburg RJ, Priori SG, Quiñones MA, Roden DM, Silka MJ, Tracy C. ACC/AHA/ESC 2006 guidelines for management of patients with ventricular arrhythmias and the prevention of sudden cardiac death - executive summary: a report of the American College of Cardiology/American Heart Association Task Force and the European Society of Cardiology Committee for Practice Guidelines (Writing Committee to Develop Guidelines for Management of Patients with Ventricular Arrhythmias and the Prevention of Sudden Cardiac Death). Eur Heart J 2006;27: 2099-2140 http://eurheartj.oxfordjournals.org/cgi/reprint/27/17/2099 